\title{
Comparison of the effects on comorbidities and the safety of gastric bypass and sleeve gastrectomy in morbid obesity patients
}

FJ Vílchez-López (1), Isabel Mateo-Gavira (1), María Belén Ojeda-Schuldt (1), María Ángeles Mayo-Osorio (2), José Manuel Pacheco-García (2), Luis Escobar-Jiménez (1), Manuel Aguilar-Diosdado (1).

(1) Endocrinology Department, Puerta del Mar Hospital, Cádiz, Spain.

(2) Surgery Department, Puerta del Mar Hospital, Cádiz, Spain.

\section{INTRODUCTION}

$\checkmark$ Obesity is a multifactorial disease associated with numerous comorbidities. Bariatric surgery is postulated as an effective tool in weight loss and improves associated pathologies.

\section{OBJECTIVES}

$\checkmark$ The aim of this study was to evaluate and compare the safety and the effects on major comorbidities associated with morbid obesity of gastric bypass (GBP) and sleeve gastrectomy (SG) 2 years after intervention.

\section{METHODS}

$\checkmark$ Cohort study with intrasubject measures (before-after) in a sample of patients with morbid obesity who underwent bariatric surgery (GBP or SG). Demographic characteristics, anthropometric parameters and cardiovascular risk factors were analyzed, at baseline and two years after surgery. Surgical complications were classified into early (first month after intervention) and late (more than one month).

\section{RESULTS}

$\checkmark 211$ patients were included. The mean age was $37.9 \pm 9.8$ years and 157 were female (74\%). The mean baseline BMI was $51.13 \pm 7.11$ $\mathrm{kg} / \mathrm{m} 2$. Regarding the surgical techniques, $178(84.35 \%)$ underwent BPG and 35 (16.4\%) SG, without significant preoperative differences between groups.

$\checkmark$ Two years after surgery, the percentage of excess weight loss was $73.06 \pm 14.06 \%$ in the BPG group vs $66.61 \pm 18.46 \%$ in SG ( $p=0.02$ ). The resolution of hypertension, dyslipidemia and diabetes occurred in $75 \%, 93.96 \%$ and $86.95 \%$ respectively in the BPG group and $63.63 \%$, $77.7 \%$ and $85.71 \%$ in the group who underwent sleeve gastrectomy. Regarding surgical complications, $15.5 \%$ had late complications in BPG group vs $3.1 \%$ in sleeve group, while the rate of early complications was $26.8 \%$ vs. $6.2 \%$ respectively $(p<0.001)$.

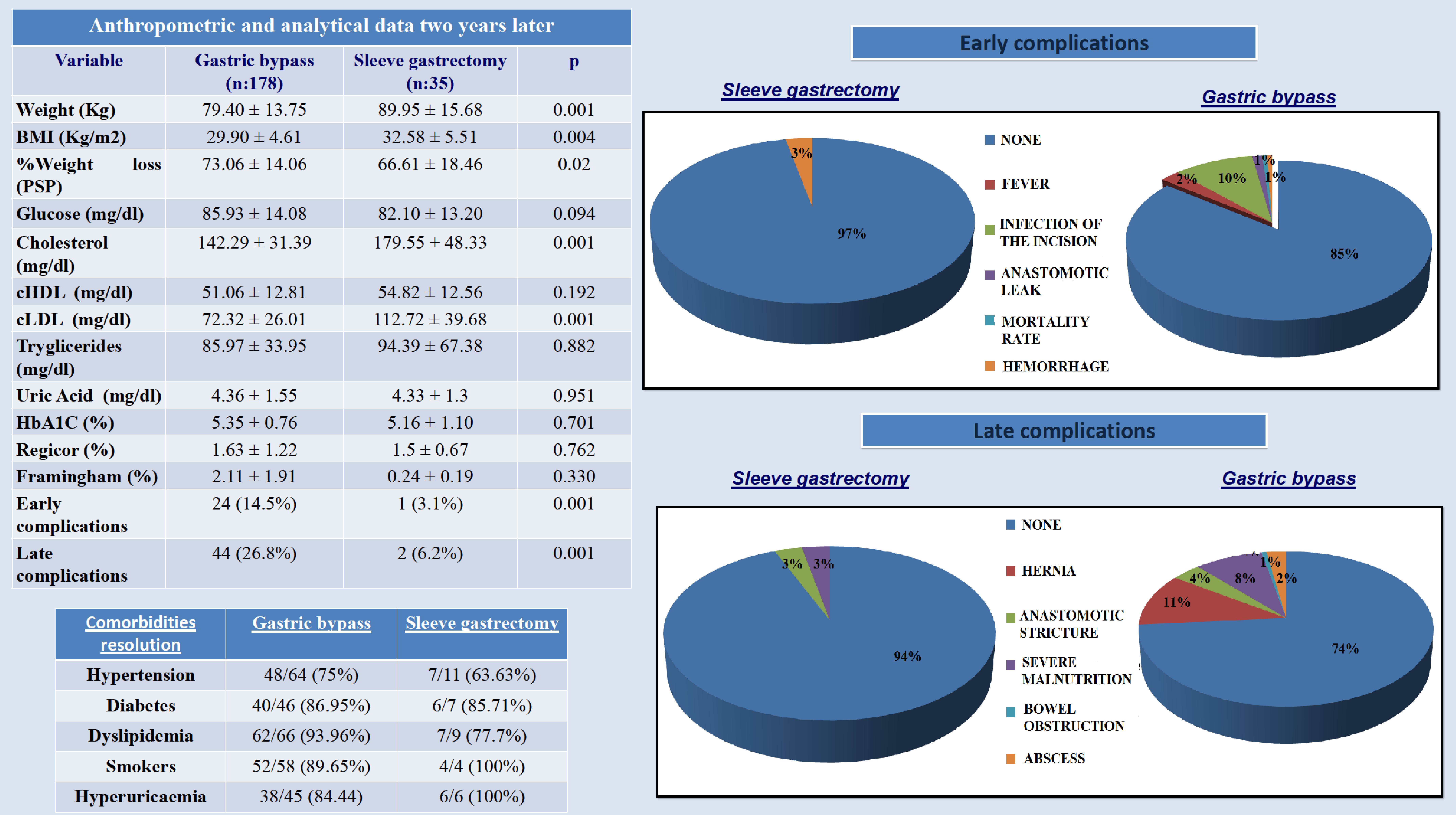

\section{CONCLUSIONS}

In our area, BPG is more effective in weight loss and resolution of dyslipidemia at 2 years, while both techniques are equally effective in resolution of diabetes and hypertension. The rate of surgical complications is lower in patients undergoing sleeve gastrectomy. 